\title{
MADMAX: A Dielectric Haloscope Experiment
}

\section{Xiaoyue $\mathrm{Li}^{a, *}$ for the MADMAX Collaboration}

${ }^{a}$ Max Planck Institute for Physics, Foehringer Ring 6, Munich, Germany

E-mail: xyli@mpp.mpg.de

Axions emerge naturally from the Peccei-Quinn (PQ) mechanism which addresses the absence of CP violation in QCD; the axions produced through the "vacuum realignment mechanism" are also a good cold dark matter (CDM) candidate. If PQ symmetry breaking had occurred after inflation, the axion mass is likely to fall in the range of $26 \mu \mathrm{eV}$ to $1 \mathrm{meV}$, which is yet to be explored experimentally.

We present a novel dielectric haloscope experiment dedicated to the direct detection of the axions that constitute the local galactic dark matter halo in the mass range of 40 to $400 \mu \mathrm{eV}$ - the MAgnetized Disc and Mirror Axion eXperiment (MADMAX). Multiple dielectric discs and a metal mirror are placed in a strong magnetic field to utilize the axion-induced coherent electromagnetic waves emitted from each disc surface and their resonances within the discs-mirror system, such that the axion-induced signal can be boosted to a level detectable by state-of-the-art low noise amplifiers. The design and sensitivity of MADMAX, ongoing R\&D activities and the project roadmap are presented.

40th International Conference on High Energy physics - ICHEP2020

July 28 - August 6, 2020

Prague, Czech Republic (virtual meeting)

${ }^{*}$ Speaker 


\section{Introduction}

In the Standard Model of particle physics, there exists a term in the QCD Lagrangian

$$
\frac{\alpha_{s}}{8 \pi} \bar{\theta} \tilde{G}_{a}^{\mu v} G_{\mu v a},
$$

which violates parity, time-reversal, and therefore $\mathrm{CP}$. As a result, a non-zero $\bar{\theta}$ leads to CP-violating observables such as the nuclear electric dipole moment (EDM). However, decades of neutron EDM measurements have set a stringent limit on the value of $\bar{\theta}: \bar{\theta}<1.3 \times 10^{-10}$ [1]. That $\bar{\theta}$ is extremely small compared to the expected value of $O(1)$ is the so-called strong $C P$ problem. Another enduring puzzle in particle physics is the nature of dark matter. Axions that naturally emerge from the solution to the strong CP problem are an ideal candidate for the cold dark matter (CDM) in the universe, which makes them a prime target for experimental searches.

There have been many haloscope experiments that aim to detect the axions in the form of CDM bound to our galaxy. The most sensitive haloscopes to date are microwave cavity based experiments such as ADMX [2], HAYSTAC [3] and CULTASK [4]. However, despite their success at the $1-10 \mu \mathrm{eV}$ mass range, such experiments run into intrinsic limits at higher axion mass due to the reduced cavity volume and Q-factor. The dielectric haloscope concept presents a promising way of detecting CDM axions in the well motivated mass range of 40 to $400 \mu \mathrm{eV}$. In this article, we will describe the theoretical motivation for searching for QCD axions in this mass range, followed by the dielectric haloscope setup, the MAgnetized Disc and Mirror Axion eXperiment (MADMAX) [5] based on the dielectric haloscope concept, and the ongoing R\&D efforts by the MADMAX collaboration.

\section{Theoretical motivation}

An elegant solution to the strong CP problem was proposed by Peccei and Quinn [6], known as the Peccei-Quinn (PQ) mechanism. A new global chiral U(1) $)_{\mathrm{PQ}}$ symmetry is introduced that spontaneously breaks at the PQ scale $f_{a} \gg \Lambda_{\mathrm{QCD}}$; the $\mathrm{U}(1)_{\mathrm{PQ}}$ symmetry is also explicitly broken by the chiral anomaly at scale $\sim \Lambda_{\mathrm{QCD}}$. Thus a pseudo-Goldstone boson - the axion, emerges from the spontaneous symmetry breaking at temperature $T \sim f_{a}$. As the temperature approaches $\Lambda_{\mathrm{QCD}}$, the axion field $\theta(x)$ starts to oscillate around the minimum of the QCD vacuum energy density $V_{\mathrm{QCD}}(\theta)$. The expectation value $\langle\theta\rangle$ is dynamically driven to zero, and thus leading to the absence of CP-violation, regardless of what the initial value $\bar{\theta}$ happens to be. This is the so-called vacuum realignment mechanism. The axion mass is given by the dynamics of the oscillation around the minimum of $V_{\mathrm{QCD}}(\theta)$. The latest calculation based on Lattice QCD yields [7]

$$
m_{a}=5.70(6)(4) \mu \mathrm{eV}\left(\frac{10^{12} \mathrm{GeV}}{f_{a}}\right)
$$

The original PQ mechanism assumed an $f_{a}$ on the same order as the electroweak symmetry breaking scale, which was quickly ruled out by beam dump experiments. Invisible axion models, most notably the KSVZ model and the DFSZ model, have a much larger $f_{a}$ and are still viable.

The axions produced by the vacuum realignment mechanism can provide the right amount of $\mathrm{CDM}$ in the universe. The allowed $f_{a}$ (or $m_{a}$ ) range which can yield the correct CDM density 
depends on the sequence of two critical cosmological events: PQ symmetry breaking and inflation. In the case where the PQ symmetry breaking occurred before inflation, axions can constitute all of the CDM for $f_{a} \gtrsim 10^{9} \mathrm{GeV}\left(m_{a} \lesssim 0.5 \mathrm{meV}\right)$, with the $f_{a}$ value depending on the initial misalignment angle at PQ symmetry breaking [8]. If the PQ symmetry breaking happened after inflation, the computation has to be based on a statistical average of the initial misalignment angle, and is complicated by the axion production via the decay of cosmic strings and domain walls. The mass range $(26 \mu \mathrm{eV}, 1 \mathrm{meV})$ is generally quoted for this scenario, with $m_{a} \sim 100 \mu \mathrm{eV}$ as the typical mass [5].

\section{Dielectric haloscope and the MADMAX setup}

In the same manner as cavity haloscopes [2-4], a dielectric haloscope also relies on the Primakoff effect [9] which converts a local CDM axion into a microwave photon in the presence of an external magnetic field; the local CDM axions can also be considered as a classical field. In a homogeneous medium in a static $B$-field, the axion field induces a static oscillating electric field

$$
\mathbf{E}_{a}(t)=-\frac{\alpha}{2 \pi \epsilon} C_{\alpha \gamma} \mathbf{B}_{e} \theta(t)
$$

where $\epsilon$ is the permittivity, $\theta(t)$ the axion field, $\alpha$ the fine structure constant, $\mathbf{B}_{e}$ the external magnetic field, and $C_{\alpha \gamma}$ the model-dependent axion-photon coupling.

$\mathbf{E}_{a}(t)$ is discontinuous at the boundary between two media with different $\epsilon$, which is not allowed by the axion-modified Maxwell equations. As a result, electromagnetic (EM) radiations are emitted at the boundary such that the continuity condition is sustained. The power emitted from a metal mirror $(\epsilon=\infty$ effectively) can be calculated as

$$
P_{0}=2.2 \times 10^{-27} \mathrm{~W}\left(\frac{A}{1 \mathrm{~m}^{2}}\right)\left(\frac{B_{e}}{10 \mathrm{~T}}\right)^{2}\left(\frac{\rho_{a}}{0.3 \mathrm{GeV} / \mathrm{cm}^{3}}\right) C_{a \gamma}^{2},
$$

where $\rho_{a}$ is the local galactic axion CDM density, and $A$ is the area of the interface. A dielectric haloscope consists of multiple dielectric discs added parallel to a mirror. In such a setup, the EM emission at selected frequencies can be enhanced by the coherent emissions from all boundaries and their constructive interference within the mirror and discs:

$$
P=P_{0} \cdot \beta^{2}(v)=1.1 \times 10^{-22} \mathrm{~W}\left(\frac{\beta^{2}(v)}{5 \times 10^{4}}\right)\left(\frac{A}{1 \mathrm{~m}^{2}}\right)\left(\frac{B_{e}}{10 \mathrm{~T}}\right)^{2}\left(\frac{\rho_{a}}{0.3 \mathrm{GeV} / \mathrm{cm}^{3}}\right) C_{a \gamma}^{2},
$$

where $\beta^{2}(v)$ is called the power boost factor $[10,11]$. The frequency and bandwidth of the boost factor can be tuned by changing the disc positions. The area under the boost factor curve $\int \mathrm{d} v \beta^{2}(v)$ is conserved, which means there is a tradeoff between the peak power and bandwidth; the integral also increases with the number of discs $N$.

Aimed at detecting QCD axions around $100 \mu \mathrm{eV}$, MADMAX proposes to use $80 \mathrm{LaAlO}_{3}$ $(\epsilon \approx 24)$ discs, each with an area of $\sim 1 \mathrm{~m}^{2}$. A sketch of the MADMAX experiment is shown in Fig. 1. The booster, focusing mirror and the antenna will be placed inside a $4 \mathrm{~K}$ cryostat. The RF part of the low noise receiver (not shown) will be placed in a different cryostat behind the antenna. The $9 \mathrm{~T}$ magnetic field will be provided by a superconducting dipole magnet, which will be situated in a separate cryostat with a warm bore of $1.35 \mathrm{~m}$ in diameter to house the booster cryostat. 


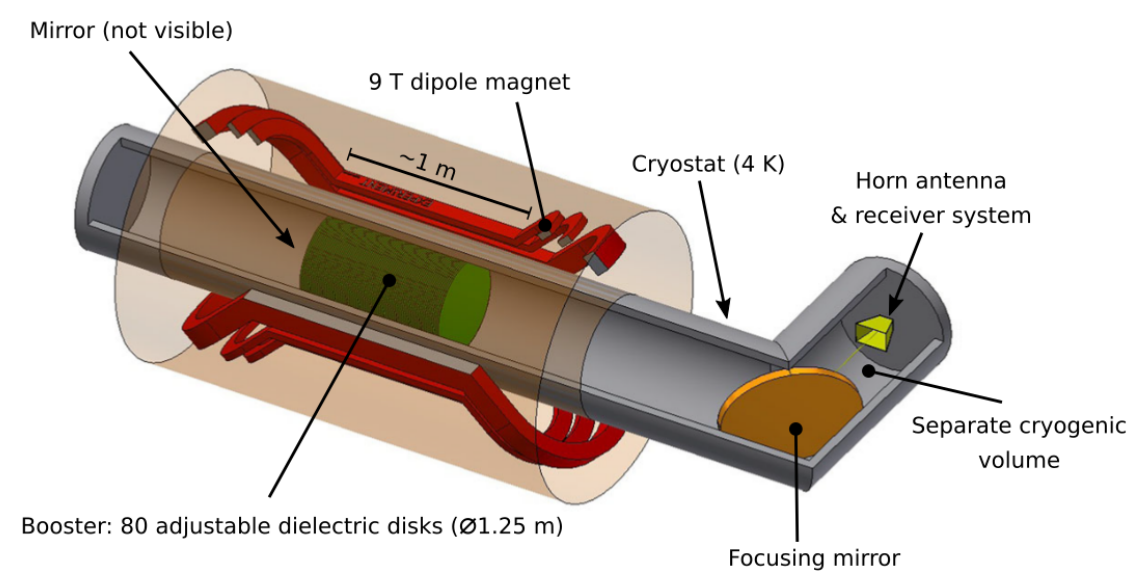

Figure 1: Conceptual sketch of the MADMAX experiment.

\section{MADMAX R\&D}

In this section, a few R\&D activities within the MADMAX collaboration are highlighted. This includes the booster simulation of finite-size, non-ideal discs, the frequency tuning procedure, the receiver chain, and disc tiling [5, 12].

\subsection{Booster simulation}

Simulations have been performed using the finite element method (FEM) as well as custom algorithms [13] to study the effects due to the finite disc size, disc tilting, surface flatness and antenna coupling efficiency. It is found that the finite disc size gives rise to modes with different dispersion relations than plane waves, which leads to an offset in the boost factor frequency compared to that of the 1D simulation. A Gaussian antenna only couples to the fundamental mode, reducing the total detected power. Other factors such as disc tilting and surface thickness variation can cause mode mixing and lead to further losses in the detectable signal power. It is estimated that for the setup shown in Fig. 1, the disc tilting should be limited to 0.1 milliradian, surface roughness $<10 \mu \mathrm{m}$, and dielectric loss $\tan \delta<\mathrm{a}$ few $\times 10^{-5}$ [12].

\subsection{Proof-of-principle setup and measurements}

Although the boost factor cannot be directly measured, it is strongly correlated with the EM response of the system and, therefore, can be inferred from the reflectivity measurement. A proofof-principle setup has been built to study the EM response and mechanical stability of the system through reflectivity measurements, as well as to develop a frequency tuning procedure. The booster of the setup is shown in Fig. 2; up to 20 sapphire $(\epsilon \approx 9.4)$ discs with $20 \mathrm{~cm}$ diameter can be moved by the precision motors. The antenna and focusing mirror used to inject the signal and measure the reflectivity are not pictured. It has been demonstrated that the system has sufficient mechanical stability, and frequency tuning with up to five discs has been achieved [14]. Fig. 3 shows an example of the measured and simulated group delay of the reflectivity.

\subsection{Receiver}

A receiver chain, which consists of a cryogenic HEMT preamplifier and three heterodyne mixing receivers, has been built and tested. The HEMT preamplifier from Low Noise Factory 


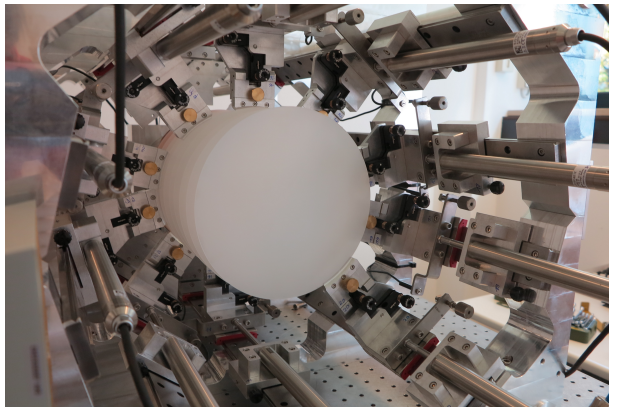

Figure 2: The proof-of-principle booster. The focusing mirror and antenna are not pictured.

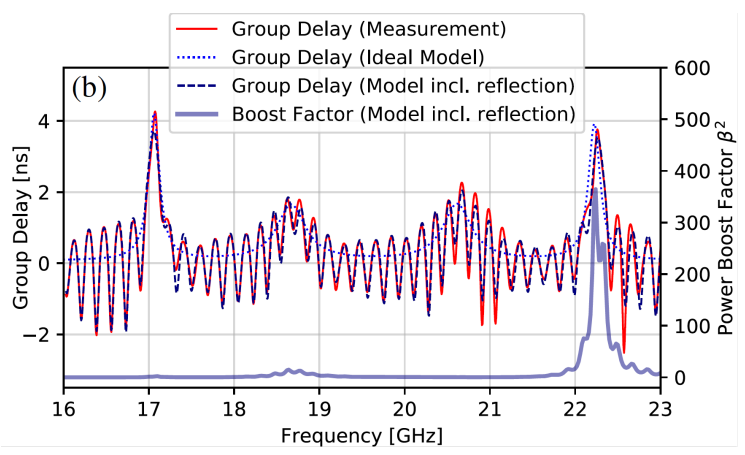

Figure 3: Group delay measurement v.s. 1D simulation of the POP setup with four discs.

has a $\sim 33 \mathrm{~dB}$ gain and $\sim 5 \mathrm{~K}$ noise temperature at $18-26 \mathrm{GHz}$ when used in liquid helium. The IF signal at the end is recorded by four samplers with $50 \mathrm{MHz}$ bandwidth connected in a daisy chain; the samplers have internal FPGAs that can perform FFT and subsequent averaging. A "fake axion" signal with power of $\sim 1.2 \times 10^{-22} \mathrm{~W}$ at $18.4 \mathrm{GHz}$ is injected into the receiver chain as a test, and it is detected with a $4.8 \sigma$ significance after two days of measurement [5]. The possibility of using a traveling wave parametric amplifier (TWPA) [15] to reduce the system noise temperature is being investigated.

\subsection{Disc tiling}

$\mathrm{LaAlO}_{3}$ is currently chosen as the material for the discs for its large dielectric constant and small $\tan \delta$ loss. However, single crystal $\mathrm{LaAlO}_{3}$ can be grown with a maximum size of 3". A tiling technology has been developed to glue small hexagon tiles with STYCAST ${ }^{\circledR}$ BLUE 2850FT to form a bigger disc. A more detailed description of the tiling technique can be found in Ref. [12]. The effect that tiling has on the booster EM properties is under study. Meanwhile, investigations of alternative disc materials which can be fabricated in larger sizes are ongoing.

\section{Project roadmap}

A prototype detector which consists of 20 tiled $\mathrm{LaAlO}_{3}$ discs with a $30 \mathrm{~cm}$ diameter will be built and commissioned at the University of Hamburg; it will serve as a testbed for technical challenges such as the disc driving mechanics. The operations of the prototype are nominally without magnetic field. In order to test its mechanical behavior in magnetic field, the prototype will also be operated inside the MORPURGO magnet at CERN that can produce a magnetic field of up to $1.6 \mathrm{~T}$, which has been approved by CERN. Should the commissioning be successful, the first competitive limits for hidden photons and ALPs around $m_{a} \sim 100 \mu \mathrm{eV}$ can be set by the MADMAX prototype by 2025.

The $9 \mathrm{~T}$ dipole magnet $\left(B^{2} \cdot A=100 \mathrm{~T}^{2} \mathrm{~m}^{2}\right)$ is the main cost and time driver of the experiment. The magnet feasibility study has been concluded with a positive assessment, and the academic and industrial partners have been appointed. The design of the magnet is currently being completed, and studies are ongoing to test the design of the superconducting coils. The projected sensitivity of the prototype and the full-scale detector is shown in Fig. 4. 


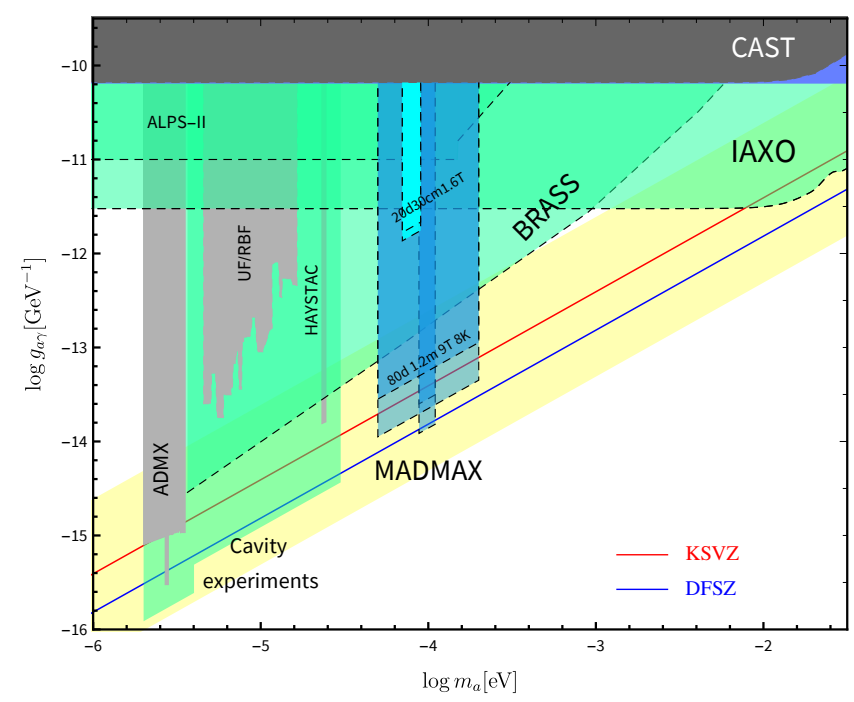

Figure 4: The axion/ALP discovery potential of MADMAX compared with two benchmark QCD axion models [12]. The excluded region (discovery potential) of other existing (future) experiments is also shown. The sensitivity range for MADMAX is shown for two cases (blue regions): a wide and a narrow mass range scan. The upper (lower) dashed lines are obtained assuming boost factor bandwidth $50 \mathrm{MHz}$ (20 MHz), 1 day (1 hour) disc realignment time, system noise temperature $8 \mathrm{~K}(4 \mathrm{~K})$, and live time of 3 years ( 5 years). $50 \%$ of the obtainable power based on 1D calculation is assumed to be detected and the signal to noise ratio is 5 .

\section{References}

[1] C. A. Baker et. al. Phys. Rev. Lett. 97, 131801 (2006)

[2] N. Du et al., Phys. Rev. Lett. 120(15), 151301 (2018).

[3] L. Zhong et al., Phys. Rev. D 97(9) 092001 (2018)

[4] W. Chung, in Proceedings, 13th Patras Workshop on Axions, WIMPs and WISPs, (PATRAS 2017): Thessaloniki, Greece, 15 May 2017 - 19, 2017 (2018) pp. 97-101.

[5] P. Brun et. al. Eur. Phys. J. C (2019) 79:186

[6] R.D. Peccei, H.R. Quinn, Phys. Rev. Lett. 38, 1440 (1977)

[7] G. Grilli di Cortona, E. Hardy, J. Pardo, G. Villadoro Vega, JHEP 1601, 034 (2016)

[8] S. Borsanyi et al., Nature 539, 69 (2016)

[9] D. A. Dicus et. al. Phys. Rev. D 18, 1829 (1978)

[10] A. Caldwell et. al. Phys. Rev. Lett. 118(9), 091801 (2017)

[11] A. Millar et. al. JCAP 1701(01), 061 (2017)

[12] S. Beurthey et. al. arXiv:2003.10894

[13] S. Knirck et. al. JCAP 08 (2019) 026

[14] J. Egge et. al. Eur. Phys. J. C 80 (2020) 392

[15] L. Planat et. al. Phys. Rev. X 10, 021021 (2020) 